\title{
Une vision $0 M S$ des facultés de médecine à travers le monde
}

\author{
Jean-Jacques GUILBER *
}

\begin{abstract}
Résumé La lecture d'un rapport, analysant des données collectées lors de la préparation de la dernière édition du «Répertoi re desfacultésde médecine » publié par I'O M S, amènel'auteur à fai re ressortir quela situation mondi ale, telle qu'el leest décritepar les institutionselles mêmes a paru suffisamment inquiétante aux en quêteurs pour les amener à faire de nombreux commentaires intéressants, questionneurs et dubitatifs. Leur diagnostic n'est pas nouveau. Les représentants des éats-membres ont voté, le plus souvent à l'unani mité, des résolutions pour y remédier dont ils n'ont pas ensuite toujours assuré la mise en application. Le secrétariat del'O M S n'a pas le pouvoir de les y forcer. Son directeur général n'et pas le gendarmedu monde dela santé. D ans les limites de sa constitution I'O M S fait ce qu'elle peut et le plus souvent ce qu'elle doit.
\end{abstract}

M ots Clés buts; sélection ; éudiants; programme ; évaluation ; responsabilité sociale; secrétariat de l'O M S.

Summary After reading a report, which analyses data collected on the occasion of the preparation of the latest edition of the "World Directory of medical schools, the author wished to show that the stuation, as described by the insttuti ons themselves, appeared sufficiently alarming to the reporters that they made many interesting and questioning comments Their diagnosis is not new. The "disinguished representatives" of the M ember States have voted, often unanimously, many Resolutions which they have not always enforced. TheW H O Secretariat does not have the vested power to forcethem to do so. Its Director General isnot the world policeman for health. Within thelimits of its Constitution WH 0 does what it can do and most often what it should do.

Key wordS aims; selection; students; programme; evaluation; social responsibility; W H O 's secretariat.

Pédagogie M édicale 2004 ; 5 : : 167-170

Le lecteur francophone de cette revue qui n'aurait pas eu I'occasion delire "A View of theWorld's M edical Schools ${ }^{1}$ 》 a ainsi évité une raison supplémentaire de se sentir déprimé par l'évolution de notre planète. Un résumé de cette enquêtelui permettra de sefairequand même uneidée de la situation des facultés de médecine à travers le monde.

Le document précité rapporte les résultats d'une analyse de données, collectées de 1996 à 1998 par un questionnaire (de 250 questions) auprès de 1657 facultés de médecine dans 159 pays et par un autre questionnaire adressé aux ministères de la santé de ces pays dans le cadre de la mise à jour de la 7e et dernière édition (2000) du
«Répertoire des facultés de médecine » périodiquement publié par l'O M S depuis plusieurs décennies.

En plus de l'analyse des données, le document est éclairé par des commentaires roboratifs suscitant la réflexion et posant de prégnantes questions. Ces commentaires contiennent de multiples "pourquoi ? ", centrés sur la problématique d'une responsabilité sociale que les facultés de médecine auraient à l'égard des communautés qu'elles sont supposées servir. C'est un questionnement auquel, malheureusement, des lecteurs universitaires « traditiona listes » peuvent d'autant moins répondre qu'ils ne se sont, semblet-il, jamais ou rarement interrogés si on en juge par leurs actions. 


\section{Buts des institutions}

L'analyse montre que $80 \%$ des répondants disent que leur institution a un exposé de ses buts (mission, mandat). La région européenne a le score le plus bas (65\%). Les buts sont exprimés dans $85 \%$ des cas sous forme de " services rendus », de "programmes de recherche » et parfois du « rôle des diplômés ». II faut noter que, sauf erreur, une tentative sérieuse de définition du profil professionnel du médecin n'a été publiée pour la première fois, en H ollande, qu'en 1994.

Le commentaire fait ressortir que nombreuses sont les facultés de médecine dont les buts ne semblent pas être en cohérence avec des activités répondant à la demande des communautés qu'elles sont supposées servir. II existerait un «manque derelation signifiante entrel'exposédes buts et un engagement objectif visà vis des problèmes prioritaires de santé de la populati on, une responsabi lité sociale ou un soutien à des problèmes de recherche susceptibles d'être au bénéfice de la majorité de la population ». Seulement $10 \%$ des budgets seraient alloués pour la recherche à des enquêtes sur les problèmes majeurs affectant la santé de $90 \%$ de la population mondiale. "Lesfacultés de médecine sont-elles de plus en plus coupées non seulement des réalités auxquelles font face leurs propres populations mai sauss d'un système de val eurs basé sur des principes de qualité et d'équité? Pourquoi sont-elles le plus souvent orientées vers des solutions technologiques concernant les maladies?».

\section{Sélection des étudiants}

L'analyse montre que classiquement la sélection est basée sur l'obtention d'un diplôme du niveau secondaire, sur un examen sélectif et/ou plus rarement sur une entrevue. Le rôle de la communauté dans le processus de sélection est rare $(22,2 \%)$. II est encore le plus rare en région européenne $(12,4 \%)$.

Le commentaire indique que cette rareté est en contradiction avec les exposés des buts des facultés, qui pour la plupart insistent sur l'importance du rôle de la communauté dans le processus de sélection. Et de poser la question : «N e serait-il pas raisonnable de s'attendre à ce que les contribuables (le budget des facultés de médecine étant couvert à $70 \%$ par des contributions publiques) aient leur mot à dire?». Q uestion courageuse !

\section{Programme des études}

Le commentaire introductif qui précèdel'analyse des données concernant ce domaine paraitra peut-être surprenant à certains lecteurs. II présuppose que « théoriquement, les facultés de médecine ont pour dessein de répondre aux besoins de santé de la population ». Surprenant ce «théorique ment», non ? I pose aussi la non moins surprenante question : « est-ce que le programme donne l'occasion aux diplômés d'acquérir des compétences pertinentes par rapport aux problèmes prioritai res de vrais individus et de vraies populations » (priorities of real people and real communities).

L'analyse indique que « la plupart des facultés offrent un programme standardisé (phase préclinique qui précède la phase clinique, enseignement principalement par confé rences magistrales, par disciplines séparées et travaux pratiques, stages dans des hôpitaux tertiaires), dont la qualité varie probablement ». La «probabilité de la variation de la qualité » des programmes d'études est dévoilée au décours de l'analyse concernant les méthodes d'enseignement.

Plus de $90 \%$ des institutions disent avoir défini des objectifs. La région européenne a le score le moins élevé. II n'est pas précisé dans le rapport s'il s'agit d'objectifs d'enseigne ment ou d'objectifs d'apprentissage mais le lecteur averti connaît la réponse.

Près de $40 \%$ des institutions conservent un enseignement par disciplines séparées.

«L'enseignement par conférences magistrales prédomine 》 (en moyenne plus de $40 \%$ du temps d'enseignement) malgré sa douteuse efficacité bien connue, autrement que pour aider l'étudiant à répéter la vul gatelocale lors des examens. L'approche dite «enseignement intégré » serait utilisée par plus de $50 \%$ des institutions et cela à divers degrés allant d'un peu à beaucoup, sans préciser non plus de quoi il sagit concrètement.

L'approche par problèmes (APP ou ARP) serait « utilisée tout au long de la phase prédinique et de la phase dinique» par moins de $30 \%$ des institutions. L'Asie du sud-est et I'Europe ont un score encore plus bas.

L'enquête ne dit rien sur les dérives éventuelles dans la mise en œuvre de l'approche par problèmes. Le lecteur averti sait qu'une dérive fréquente est que, contrairement à l'idée originale de I'APP, cene sont pas les étudiants qui, en fonction d'un problème (choisi par les enseignants ou identifié en milieu professionnel par des étudiants), définissent leurs objectifs d'apprentissage, mais que ce sont les enseignants qui le font (objectifs d'enseignement). II en résulte que les étudiants se contentent d'apprendre la 
théorie correspondante sachant qu'elle sera à la base de l'examen. Concernant cette dérive "classique », un vice doyen chargé de la formation me confiait récemment que "Malheureusement c'et comme cela pratiquement depuis le début. Originellement les dossiers remis aux étudiants ne contenaient pas les objectifs. Le stress des examens a fait qu'ils ont fini par obtenir les dossiers des tuteurs qui contenaient lesdits objectifs et qui ensuite ont circulé en sousmain. On a donc fini par mettre les objectifs dans les dossiers des étudiants».

L'orientation vers les problèmes de santé de l'individu occupe 6,5 fois plus d'heures d'enseignement que les problèmes de santé de la population (médecine générale, médecine communautaire, épidémiologie, etc.) et moins de $10 \%$ du temps d'enseignement. M oins de $2 \%$ desinstitutions incluent une formation pratique en gériatrie alors que la population âgée augmente.

L'analyse montre « un faible intérêt pour les initiatives du domaine de la santé publique basées sur des facteurs orientés vers les besoins de la communauté(population) et qui ont un impact sur la durée et sur la qualité de vie». On pourrait espérer que cet intérêt se manifeste à l'occasion de cours dont les titres «culture», «philosophie » ou « social » permettraient à l'étudiant de ne pas être confiné au domaine bio-mécanique. $M$ alheureusement, ces sujets sont traités au cours de conférences magistrales avec un impact quasiment nul.

Le commentaire fait remarquer qu'« il n'y a pas de mériteà bien enseigner quelque chose hors propos (effectivement pourquoi chercher à automatiser un piège à souris si le but est d'attraper un éléphant) et réciproquement il n'y a aucun avantage à mal enseigner quelque chose de pertinent » (malheureusement c'est trop souvent le cas).

\section{Evaluation de la performance des diplômés}

Là, cher lecteur, on entre dans le vague et l'ambigu. L'analyse des données indique que «virtuellement toutes les facultés de médecine utilisent " certains standards » (étalons de qualité) pour évaluer la performance des étudiants ». "La plupart des institutions utilisent un mélange d'épreuves écrites, pratiques, orales et d'épreuves d'observation en milieu professionnel ». Des épreuves écrites sont utilisées dans $96 \%$ des cas pour évaluer les «connaissances » et entre 61 et $73 \%$ pour évaluer le « raisonnement ». Les « attitudes et l'aptitude à communiquer » seraient évaluées par des épreuves en milieu professionnel.
Le commentaire suggère que « en théorie, une évaluation objective (unbiased) de la « capacité » d'un étudiant à se comporter en médecin « compétent » devrait être une indication raisonnabledel'eficacitéd'unefacultéde médecine. Cependant un tel type d'évaluation ne se rencontre pas nécessairement 》 (?) «Les critères utilisés peuvent ou non correspondre à des compétences reliées à des problèmes de santé importants ». C'est grave, docteur ?

Le commentaire ose même laisser entendre qu' «il ya un problème majeur ». «II n'y a pas d'étalon (yardstick) pour évaluer la compétence médicale.. II y a besoin de « mieux (?) définir les attributs et qualités requises de la part d'un médecin... pour affronter les problèmes de santé importants», sinon les institutions risquent de ne pas bien former les étudiants et même de ne pas s'en rendre compte, comme aurait dit le sublime R.F. M ager.

Le mot « validité » (degré avec lequel un instrument mesure cequ'il a pour objet de mesurer) n'apparaît nulle part, ni dans l'analyse des données ni dans les commentaires. C'est pourtant bien de cela qu'il s'agit, mais c'est un sujet qui fâche.

\section{Commentaire}

II est clair que l'analyse des données mentionnées dans cet excellent rapport ne permettait pas, selon le commentaire, de répondre aux questions suivantes : "quel nombre de facultés peut être jugé comme « satisfaisant ?» ; « chaque pays devrait-il avoir une faculté de médecine?»; "quelles sont les «meilleures» facultés de médecine?»; « au lieu d'augmenter le nombre de facultés de médecine, un pays ne devrait-il pas plutôt ouvrir d'autres institutions de formation permettant d'améliorer la qualité des soins à la population?». Ce silence ne me semble pas trop grave.

En revanche, comme il est dit que le but de l'enquête était plutôt de « collecter des données de base permettant d'estimer dans quelle mesure les facultés de médecine remplissent leur rôle en réponse aux besoins de santé des individus et des collectivités", le lecteur réalisera que le flou artistique qui entoure le domaine de l'évaluation des compétences professionnelles risque de ne pas permettre de savoir si le but de l'enquête avait une chance d'être atteint.

Le commentaire demande, sans malheureusement pouvoir y répondre : " considérant que ces facultés sont coûteuses à créer, à entretenir et à améliorer, répondent elles à leur mission et la société en a-t-elle pour son argent ? ». 
Le rapport ne permet pas non plus « de faire un rapport entre le nombre et la distribution des facultés de médecine d'une part et le niveau général de santé des populations concernées ». C ela est plus grave.

Lorsque, le 7 décembre 1999, I'Institut de médecine (des Etats-Unis) avait informé le Président Clinton, que «98 000 américains perdent la vie chaque année suite à des erreurs médicales évitables», il avait superbement répondu « N ous avons le meilleur système de soins de santé du monde, les meilleurs professionnels pour dispenser cessoins ». $M$ ême si le nombre de morts précité aurait dû permettre au Président des Etats-U nis d'avoir un doute, il avait peutêtre raison comme il avait peut-être tort. En vérité personne n'en sait rien en raison du niveau douteux de validité des épreuves conduisant au diplôme de méde$\mathbf{c i n}^{2}$. Et ne pas savoir cela est important, grave, sérieux et tragique.

\section{Mais alors, que fait l'OMS ? Eh bien, l'OMS fait ce qu'elle peut et le plus souvent ce qu'elle doit}

Les fonctionnaires du secrétariat de l'O M S « fonctionnent ", les colloques " colloquent», les experts «conseillent » et chacun va, entre deux voyages en mission, de réunion de comité en réunion de groupe de travail, suivis de conférences avec publication d'épais rapports et ensuite les assemblées générales accumulent résolutions sur résolutions comme si dire égalait faire.

Premier problème : les états-membres mettent ou ne mettent pas en application les résolutions que leurs représentants ont votées. Exemple : le rapport analysé dans cet article présente, dans ses commentaires, d'excellentes recommandations visant la qualité des soins par l'amélioration des compétences professionnelles des professionnels de la santé. Or, ces recommandations sont en ligne directe avec une résolution (Résolution $A$ 29/15) présentée il y a plus d'un quart de siècle par Tamas Fülöp et adoptée officiellement en mai 1976 par I'Assemblée générale. Sa mise en application par les états- membres n'a guère suivi. Le secrétariat de l'O M S n'a pas le pouvoir de les y forcer.

D euxième problème : il est admirable que le budget alimenté grâce aux contributions des pays les plus riches soit distribué aux pays les plus pauvres. M ais, si la corruption s'en mêle au niveau local, le secrétariat de I'OM S n'a aucun moyen constitutionnel d'y remédier. Son directeur général n'est pas le gendarme du monde de la santé. Un journaliste suisse ${ }^{3}$ me rétorqua que «s s vraiment seule une petite partie des sommes distribuées par les organisations internationales arrive jusqu'aux hôpitaux et aux patients du tiersmonde parce que l'argent a fini dans les poches de ministres et de fonctionnaires, alors il faut prier ces organisations d'utiliser d'autres canaux pour promouvoir la santé dans les pays pauvres, sinon il ne sagit plus d'aide mais de complicité ». C e point de vue, de la part d'un professionnel réputé, montre combien le rôle du secrétariat et la constitution de I'O M S sont mal connus.

\section{Références}

1. Boelen Ch, Boyer M H. A View of theWorld's M edical Schools. Defining new roles 2001 [O $n$-line]. Disponible sur : www.network.unimaasnl/miscellaneousindex.htm

2. Guilbert JJ." Nous avons les meilleurs médeans du monde » C'est peut-être vrai mais pourriez vous le prouver? Pédagogie M édicale Novembre $2002 ; 3$ : 210-211.

3. Guilbert JJ. Les accusations du bon Dr Beat. A propos d'un documentaire de Temps Présent, Tribune, M édecine\& H ygiène 2004, n² 2481, 987. 